\title{
Technical and Economical Merits of Power Systems Interconnection
}

\author{
Abdullah M. Al-Shaalan \\ Electrical Engineering Department, College of Engineering, King Saud University, Riyadh, KSA. \\ Email: shaalan@ksu.edu.sa \\ Received August $13^{\text {th }}, 2013$; revised September $12^{\text {th }}, 2013$; accepted September $20^{\text {th }}, 2013$ \\ Copyright (c) 2013 Abdullah M. Al-Shaalan. This is an open access article distributed under the Creative Commons Attribution \\ License, which permits unrestricted use, distribution, and reproduction in any medium, provided the original work is properly \\ cited.
}

\begin{abstract}
This work aims at exploring the effects of the interconnection between isolated electric power systems upon some important aspects such as enhancing reliability levels along with reducing installation and operation costs. To discern the advantages associated with this study, the developed methodology has been applied to three existing power systems in the northern region of the Kingdom of Saudi Arabia presently within the concession domain of the Saudi Electric Company (SEC). These systems have been established to meet the present loads and to withstand future electrical demands for a period of time before additional generation and transmission reinforcements are needed. In this work, reliability measures have been utilized to determine the period that these systems can fulfill the present and future loads without affecting the reliability levels and the threshold that an additional capacity should be added to maintain those required reliability levels. In application to the reliability criteria, technical, operational and economic advantages can be realized, i.e., higher reliability levels and lower installation and operation costs after the proposed interconnection between these selected isolated power systems take place.
\end{abstract}

Keywords: Reliability Index; Power System; Cost; Interconnection

\section{Introduction}

Interconnection of electrical power systems is an effective means of not only enhancing the overall system reliability but also reducing its operating reserve. The diversity existing between different systems in regard to their load requirements and capacity outages will allow the systems to assist each other in times of emergencies and generation deficiencies. Also, it will permit systems to operate in less reserve than what would be required for being isolated at a given risk level. The benefits that may accrue from systems interconnection depend mainly upon the operating reserves in the individual systems, their outage rates, the tie-line capacity and the type of agreement among the systems regarding the size of the emergency assistance.

From the preceding section, it is evident that reliability is one of the most important criteria which must be taken into consideration during all phases of power system planning, design and operation. Reliability criterion is required to establish target reliability levels and to, consistently, analyze and compare the future reliability levels with feasible alternative expansion plans. This need has resulted in the development and applications of reliability evaluation and modeling methodologies.

In power system reliability evaluation, the most widely used reliability index by electric companies is the Loss of Load Expectation (LOLE). This index can be used for specifying the appropriate timing for future capacity additions as well as for comparing between various alternative expansion plans. It indicates the average power outages time accumulated in a specified period (for example, number of days in one year) due to capacity deficit. This research will, also, demonstrate other complementary reliability indices that are-to the author's knowledgegiven less consideration in present realistic and applied cases. These indices are the "Expected Demand Not Served (EDNS)", the "Expected Energy Not Served (EENS)" and the "Energy Index of Reliability (EIR)".

\section{Survey to Some Existing Works on Power Systems Interconnection}

In this section, an overview of some existing works is attempted to get acquaintance with other countries' experiences and practices in systems interconnections. The 
author in [1] proposed a flexible AC power transmission link technology for linking two asynchronous independent power systems. The proposed flexible asynchronous ac link (FASAL) system essentially consists of a rotating transformer which is put in the ac tie-line between two separate power systems or grids. The direction and the magnitude of power flow are controlled by controlling voltage and/or frequency. Simulink model of proposed FASAL system has been developed for the analytical study and the result verifies the power transfer capability of the proposed system.

In Reference [2], the author suggested that for evaluation of the synchronous interconnection of the African regional power pools interconnection, security issues should be taken care of before any economic analysis is done. Power system security studies have been used in industries only at the time of planning. This paper also addresses a future real time security system in operation of liberated power pools.

In [3], the author stated the advantages, requirements and problems arising from the interconnection of electric power systems in the Arab world. He also presented the planning principles for grid systems, procedures for power system development planning, technical aspects of interconnection and possibilities of increasing interconnection capacity by limited means. The results of studies investigating the feasibility of interconnecting the power networks of the six countries, which form the Gulf Cooperation Council (GCC), were presented. The author also showed existing and future interconnections of power systems in the Arab world.

In [4], the author emphasized the need, especially in developing countries, for consolidating the dispersed electric utilities in the isolated regions as a prerequisite for future interconnecting these regions via local national grids and with other neighboring countries.

In [5], the author presented methodologies and techniques that can be adopted and used in the quantitative assessment of power system reliability and its application to the cost/benefit evaluation in system generation expansion planning particularly for interconnected power systems.

In [6], the authors proposed a PMU (phasor measurement unit) based on monitoring and estimation scheme of power system small-signal stability in Singapore-Malaysia interconnection power system through a $50 \mathrm{~Hz}$ and $500 \mathrm{kV}$ transmission line. Two PMUs are installed in the power system interconnection network of SingaporeMalaysia. One PMU is located in Singapore and the other one is in Malaysia (Penang). Both PMUs measure the single-phase voltage phasor. The data filtering technique based on Fast Fourier Transform (FFT) was employed to extract oscillation data for single mode. Finally, some analysis results of monitoring and estimation of
Singapore-Malaysia interconnected power system based on application practice of the Campus-WAMS were presented and analyzed.

\section{The Developed Methodology for the Proposed Study}

For the purpose of this study, a computer program has been developed (Figure 1) which can model and simulate the methodologies and techniques adopted and demonstrated in this work. The following steps describe the principal operations of this developed computer program.

\subsection{When Systems Are Isolated}

1) Specifying the data relevant to each system under study.

2) Building the Capacity Outage Probability Table $(C O P T)$ for each system, using the Forced Outage Rates (FOR's) pertinent to each generating unit in each system.

3) Convolving the COPT with the Load Duration Curve $(L D C)$ for each individual system.

4) Evaluating the risk index level "Loss-of-Load-Expectation (LOLEe)" for each individual system and then comparing it with the risk index level prescribed by the Management ( $L O L E p$ ).

5) If the LOLEe exceeds the LOLEp, extra unit(s) must be added to the existing system capacity until the risk index level, prescribed by the management, is satisfied, otherwise proceed to the next year and repeat the same process with the new forecasted load.

6) Evaluating other complementary and essential indices such as the Expected Demand Not Served (EDNS), Expected Energy Not Served (EENS) and the Energy Index of Reliability (EIR). These indices are used as evident indicators to enable the system planner to discern between the planning outcomes and hence select the most reliable and least cost among them. These indices are utilized to vindicate the economic and technical merits of interconnecting power systems rather being dispersed and isolated.

7) Assessing and estimating the overall system cost based on the added generating unit(s) to the system at every stage of the planning horizon.

\subsection{When Systems Are Interconnected}

1) Specifying the data relevant to each system under study.

2) Building the Capacity Outage Probability Table $(C O P T)$ for the combined systems (i.e. combine capacity states with their associated probabilities).

3) Repeating the above mentioned steps (c-g). The capacity assistance $C_{T}$ is a capacity which can be transferred through the tie-line and added to the existing 


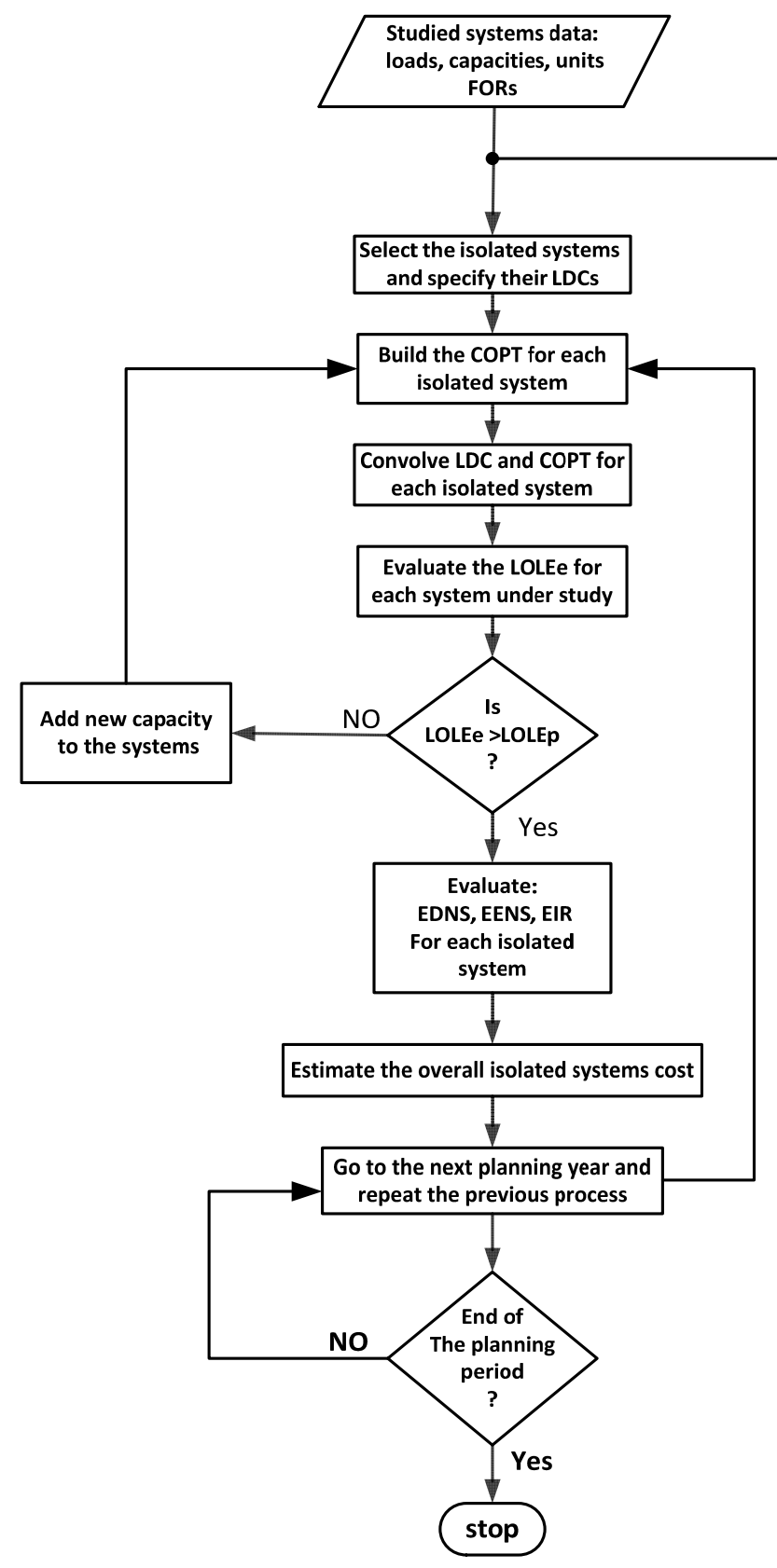

(a)

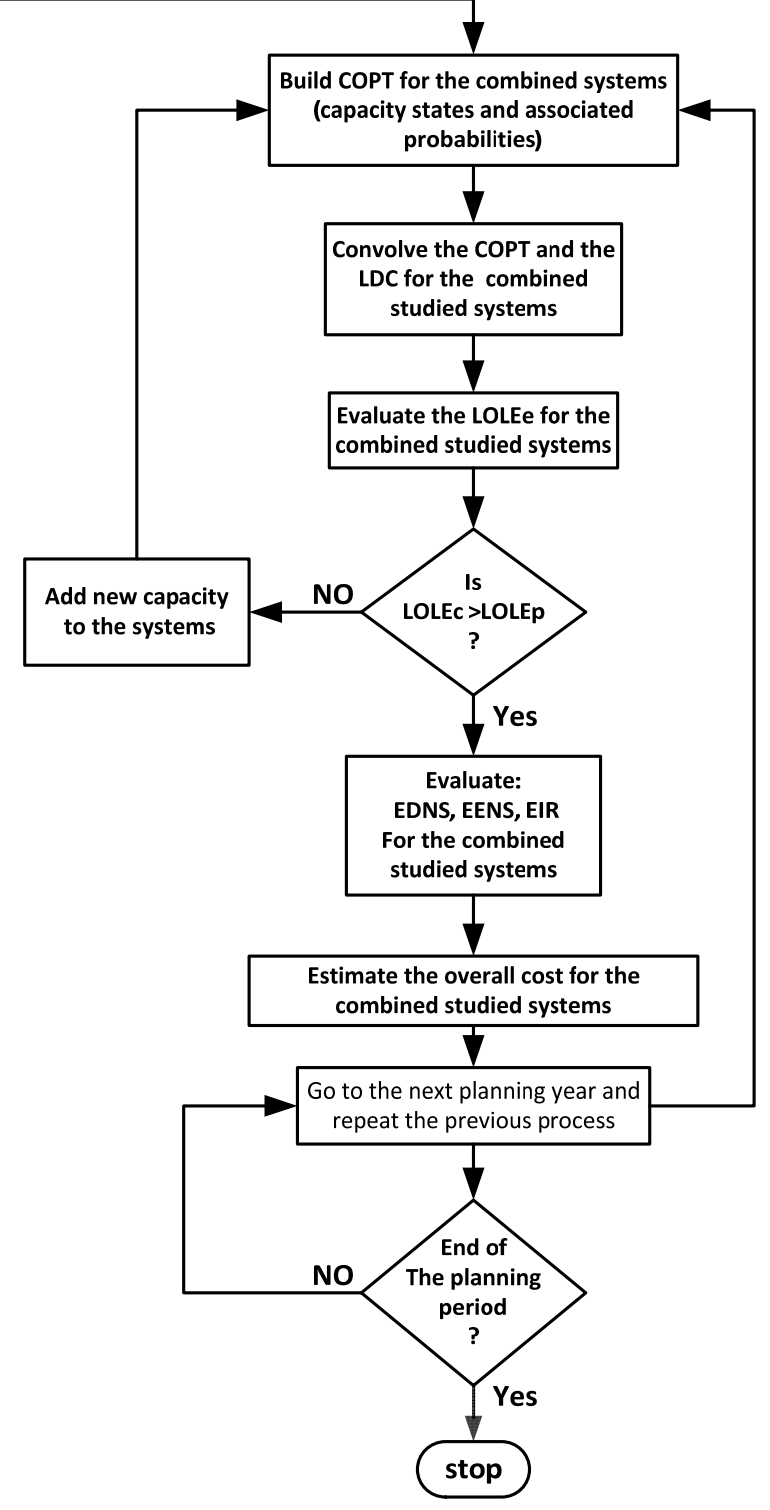

(b)

Figure 1. Flowchart processes of the adopted computational algorithm. (a) Isolated systems; (b) Interconnected systems.

capacity model of the assisted system. For the evaluation of the risk index level, the assisted system proceeds as in the case of the isolated system risk index evaluation.

\section{Application of the Developed Methodology to Real-Case Systems}

The previous modeling techniques have been substantiated and then implemented on real and practical electric power systems serving three large cities in the northern region of the Kingdom of Saudi Arabia, namely, Hail,
Jouf and Tabouk and designated in this study as (A), (B) and (C) respectively. These cities are characterized by high population density, multiple of governmental, industrial and agricultural projects, so, high future demand is anticipated. Recently, the existing power systems in these cities have been transferred to the domain of the Saudi Electric Company (SEC) that has been established in 1999.

The generation expansion planning considered for this study spans over the next eight years (2012-2020) to specify the timings of capacity additions and to determine 
the reliability levels for each system before and after the proposed interconnection. The target is to explore and investigate the technical and economic merits that might ensue as a result of systems interconnection.

\subsection{Technical Advantages of Systems Interconnection}

The aim of this study is to specify the reliability levels for each system individually as a result of future load growth over the next eight years (with the assumption of no units addition to the system) and the expected deterioration of reliability levels as a result of diminishing reserve and capacity deficit. After specifying the year that reliability level has exceeded the prescribed reliability level, capacity addition (new generating units) can be decided upon or interconnection with another system can be an optional solution.

Figure 2 shows the results of the study that has been conducted on the three systems: (A), (B) and (C) to investigate their reliability levels as being isolated over the next eight years using the LOLE criterion. It is clear from the figure that if the required level of the LOLE is set at 0.1 days/year, all systems will exceed that prescribed risk level. Therefore, new generating units must be added to each system to improve their risk levels and avoid power outages and service interruptions.

After adding concept of systems interconnection, the study has been conducted and the results are shown in Figure 2 where it can be realized that reliability levels have been improved after system interconnection. For instance, system (A) will need no capacity addition until the year 2014, and with respect to systems (B) and(C) they will exceed their reliability limits at years 2015 and 2016 respectively.

To demonstrate and ensure the benefits of intercom- nection between systems in enhancing and improving their reliability levels, the effect of interconnection upon other complementary reliability indices stated previously, i.e. EDNS, EENS and EIR has been investigated. System (A), being the largest among the three systems, has been selected for this demonstration. Figure 3 shows the results of the investigation that declares to what extent the size of the expected demand not served (EDNS) has been reduced after systems interconnection. Figure 4 also shows the reduction in the expected energy not served (EENS) due to the effect of interconnection. The EIR index was also investigated and the results are shown in Figure 5 where it is obvious from the figure that the energy loss has decreased and reliability indicator has risen to the better level after interconnection between the systems.

\subsection{Economic Advantages of Systems Interconnection}

The purpose of this part of this study is to assess the economic advantages that may yield as a result of interconnection between the above mentioned three systems. A study, which spans for eight years (2012-2020), has been conducted. The LOLE index has been specified for the three systems and set at $0.1 \mathrm{~d} / \mathrm{y}$ and to be constant over that selected planning period (this number is considered to be reasonable as being adopted in industrial as well as fast developing countries). The study was concerned about that threshold year that reliability will deteriorate (i.e. LOLE exceeds the prescribed specified limit) as a result of future load growth. To correct the risk level and maintain it within the prescribed limit set by the management, additional generating units must be added (in case of system being isolated) or should be interconnected with other system(s) after the latter is reinforced

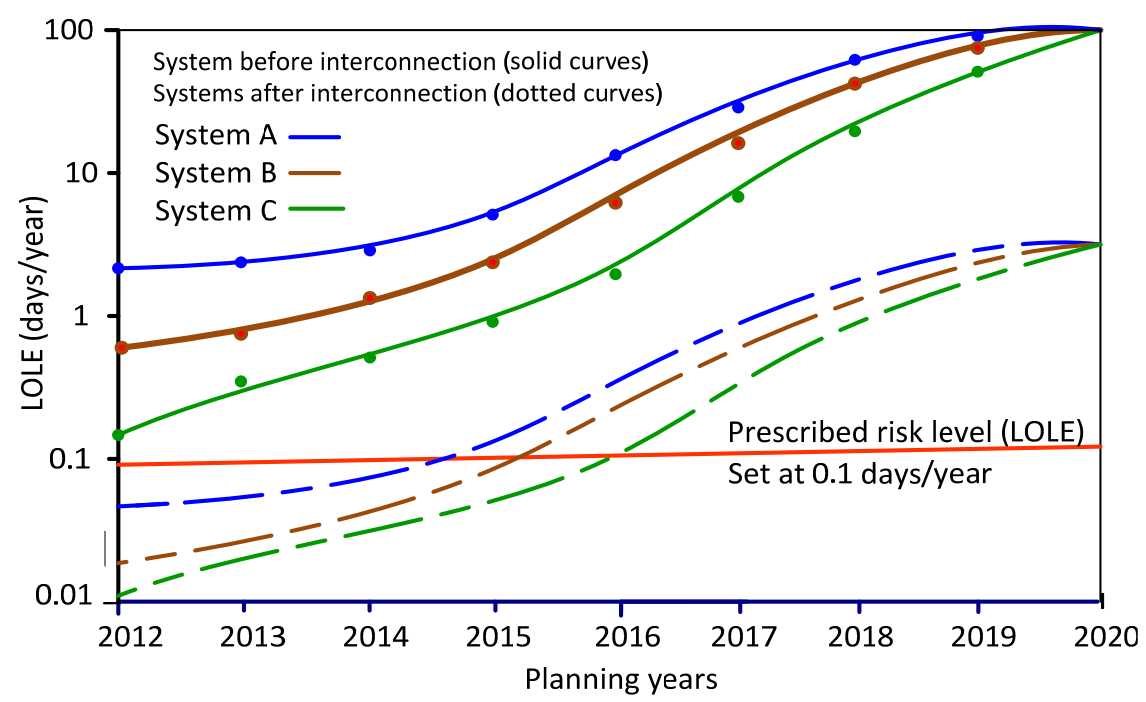

Figure 2. Systems LOLE levels before and after interconnection. 


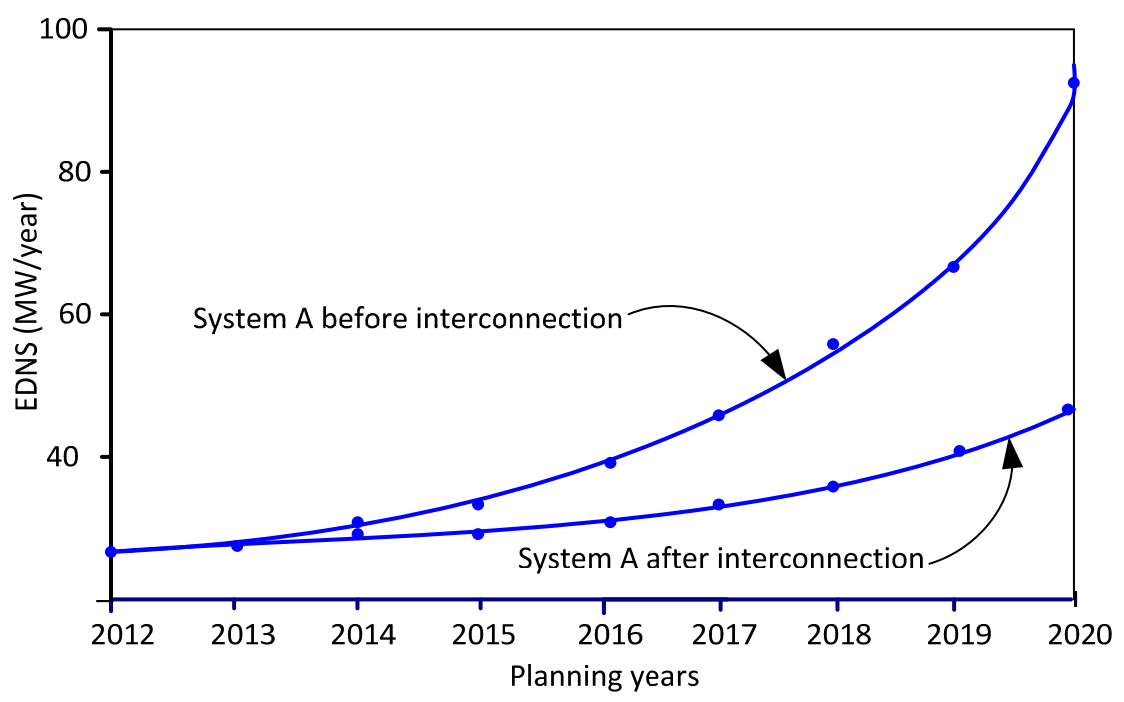

Figure 3. EDNS before and after interconnection.

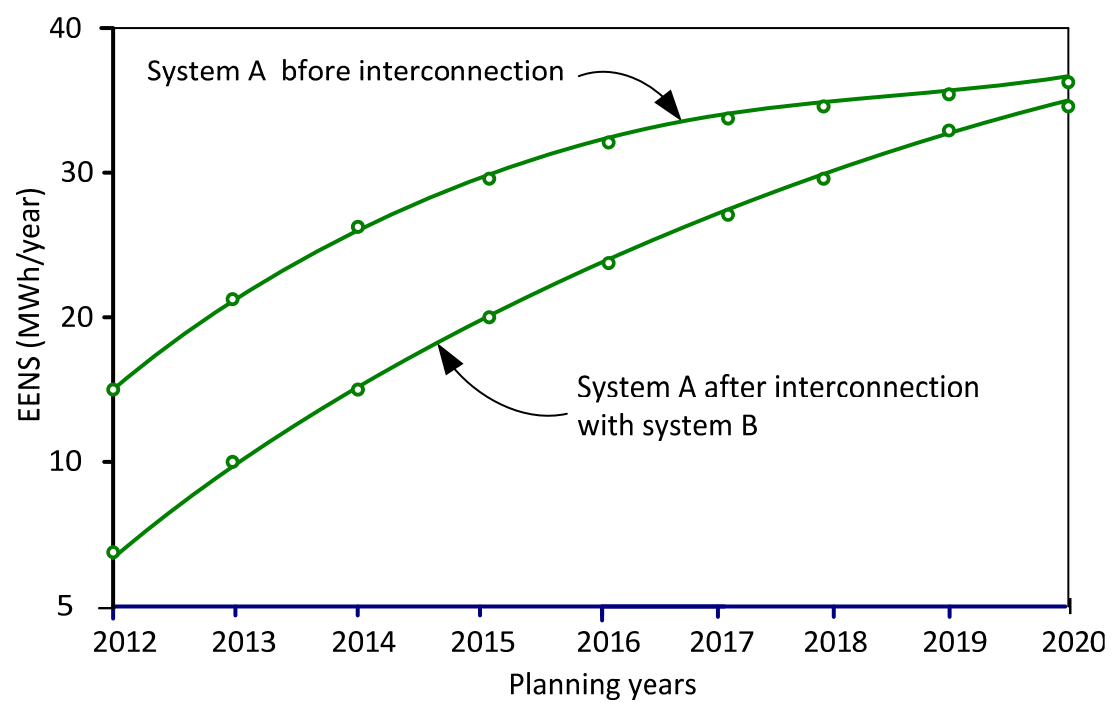

Figure 4. EENS before and after interconnection.

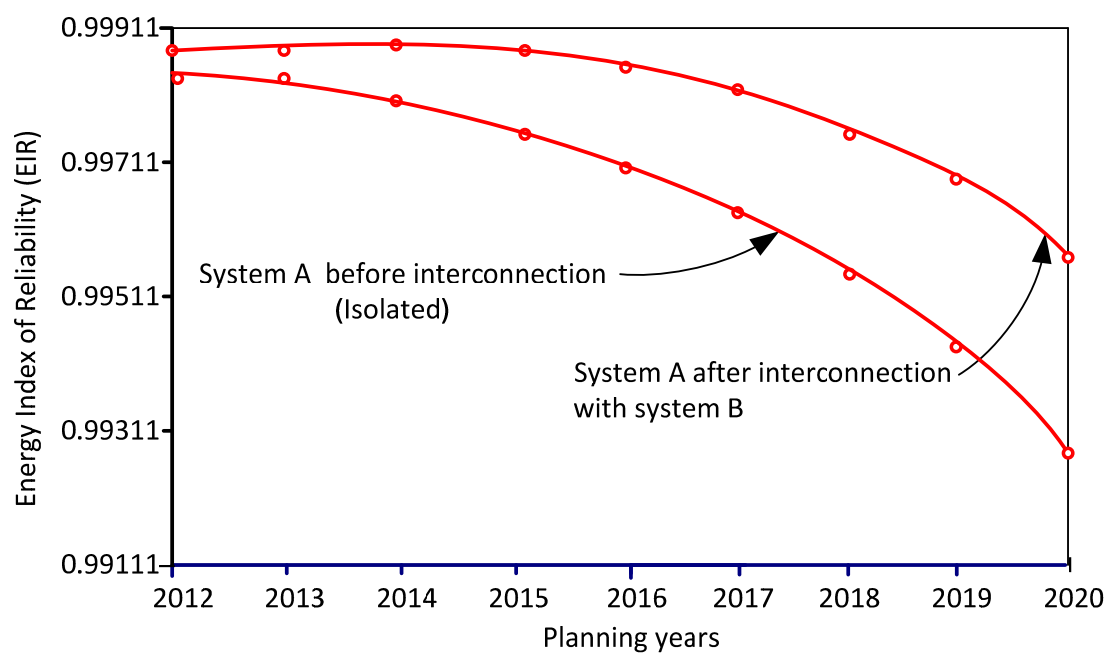

Figure 5. EIR before and after interconnection. 
by new generating units. For estimating the cost of the added units before and after interconnection, the processes shown and described in Section 4 have been utilized. Figure 6 portrays the findings of this study where they postulate that each system will benefit from being interconnected and there will be substantial savings [in Million USD (MUSD)] in both capital (fixed) and operating (variable) costs. This can be estimated as 56\%, 50\%, and $60 \%$ for systems (A), (B) and (C) [i.e. Hail, Jouf and Tabouk] respectively. Furthermore, there will be an improvement in the three systems reliability levels as analyzed and discussed in the preceding section.

\subsection{Optimal Reliability Level}

Evaluation of optimal reliability levels is a major step in power system planning process to ensure continuous and quality service with reasonable cost. The system planners perform sensitivity analysis based on economic variations, installation and transmission costs. Therefore, LOLE reliability index has been applied for system (A) electric system using the economic concepts and the reliability criterion shown in Type equation here [4,5]. In the analysis, new generating units of $68 \mathrm{MW}$ (identical to the present installed units in system A) have been added to the system when reliability levels deteriorate below the prescribed level. To arrive at the most appropriate range of reliability levels, system cost (SC) has been weighted with the outages cost (OC). System cost includes unit installation cost as well as the fuel and maintenance cost. Outages cost represents the cost of losses suffered by the society (all classes of customers) due to insufficient capacity and consequently, energy curtail- ment. The total system cost (TSC) depicts the overall cost endured by the customers in return of power supply and its availability.

In an attempt to arrive at the most optimal reliability level that ensure the least system cost, the above mentioned costs have been investigated employing system (A). The results of the investigations are illustrated by Figure 7 where it manifests that system cost (SC) increases as reliability level increases but the outage cost (OC) decreases as a result of reliability improvement due to more system investment and adequate generating capacity additions. The most optimal range of reliability levels, as depicted by the figure, varies between 0.338 and 0.675 days/year. However, in some cases adding new capacity may not signify the ideal solution to meet increasing future loads and maintain better reliability levels. Therefore, it is better to enhance operating unit's performance through regular preventive maintenance. Also, it is an imperative to establish a good co-operation between the supply side (electric company) and the demand side (the consumers) through well-coordinated load management strategies, improving system load factor and correction of power factor. Hence, system will be capable of meeting loads efficiently and reliably particularly in power system interconnection.

\section{Conclusion}

In this study, reliability and costs criteria have been established and applied for power systems planning and interconnection. To substantiate the developed methodology, three electric power systems in the northern region of the Kingdom of Saudi Arabia have been selected as a

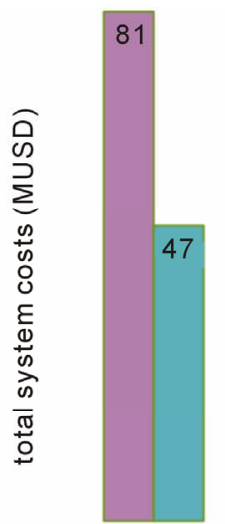

(A)

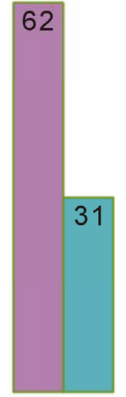

(B)

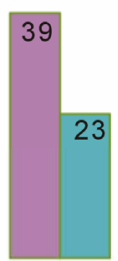

(C)

\begin{tabular}{|l|c|c|c|}
\hline Units (isol) & 2 & 4 & 3 \\
\hline Costs (isol) & 23 & 16 & 10 \\
\hline Units (inter) & 1 & 2 & 1 \\
\hline Costs (inter) & 13 & 8 & 6 \\
\hline
\end{tabular}

Figure 6. Cost (MUS\$) for isolated and interconnected systems. 


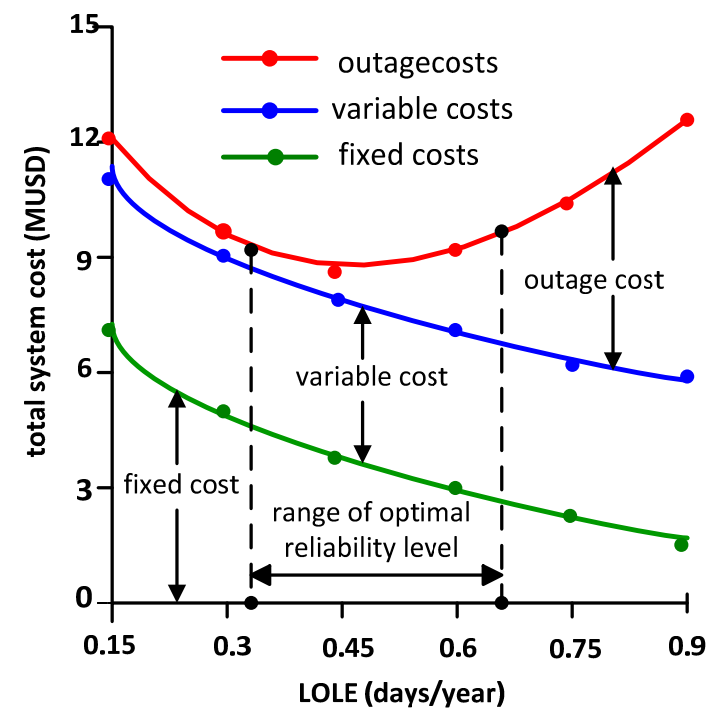

Figure 7. Variation of reliability levels with total system cost.

model for this study. These systems, at the present time, are isolated, dispersed and subjected to random failures. The results display the advantages and benefits that may accrue from systems interconnection in upgrading reliability levels, reducing systems cost, limiting service cease and mitigating energy interruptions.

\section{Acknowledgements}

The Author thanks Al-Zamil Chair for Electricity Con- servation, College of Engineering, King Saud University for supporting this work.

\section{REFERENCES}

[1] I. Imdadullah, "Flexible Asynchronous ac Link for Power System Network Interconnection,” IEEE Poly-Tech Conference on Electrical Engineering Section, Cleveland, 29-31 May 2012, pp. 1-6.

[2] M.-S. Chen, "Security Issues of Power System Interconnection,” IEEE Power Engineering Society, General Meeting, 12-16 June 2005, Vol. 2, pp. 1797-1800.

[3] E. S. Ibrahim, "Interconnection of Electric Power Systems in the Arab World,” IEEE Power Engineering Journal, Vol. 10, No. 3, 1996, pp. 121-127. doi:10.1049/pe:19960303

[4] A. M. Shaalan, "Essential Aspects of Power System Planning in Developing Countries," Journal of King Saud, Engineering Science, Vol. 23, No. 1, 2011, pp. 28-32.

[5] A. M. Shaalan, "Reliability Evaluation in Generation Expansion Planning Based on the Expected Energy Not Served,” Journal of King Saud, Engineering Science, Vol. 24, No. 1, 2012, pp. 11-18.

[6] D. Despa, Y. Mitani, L. Changsong and M. Watanabe, "PMU Based Monitoring and Estimation of Interconnected Power Oscillation for Singapore-Malaysia Interconnection Power Systems," IEEE Conference on Power Engineering Society General Proceedings, Singapore City, 27-29 October 2010, pp. 476-480. 